\title{
Statin withdrawal and treating COVID-19 patients
}

\section{David S. Fedson (1)}

Sergy Haut, France

Correspondence

David S. Fedson, 57 chemin du Lavoir, 01630 Sergy Haut, France.

Email: davidsfedson@gmail.com

\section{Funding information}

This study received no support from any external funding organization.

\begin{abstract}
Most but not all observational studies of statin treatment of COVID-19 patients suggest that treatment improves outcomes. However, almost all of these studies fail to consider that withdrawing statins after hospital admission may have detrimental effects, a finding which cardiovascular investigators have known for 15-20 years. Continuing or starting statin treatment after hospital admission consistently improves cardiovascular outcomes. Similarly, inpatient statin treatment of COVID-19 improves survival. For this reason, observational studies of the effectiveness of outpatientdocumented statin treatment of COVID-19 patients must consider the negative consequences of statin withdrawal after hospital admission.

KEYWORDS

COVID-19, mortality, statin treatment, statin withdrawal
\end{abstract}

\section{1 | INTRODUCTION}

The COVID-19 pandemic has caused worldwide social, economic, and political disruption. Its pathogenesis is largely due to extensive disruption of innate and adaptive immunity, altered interferon responses, and coagulation abnormalities that lead to microvascular immunothrombosis. Many of these changes are caused by endothelial dysfunction. ${ }^{1}$

Antiviral treatments for COVID-19 have been only marginally effective. Antibody treatments (convalescent plasma and monoclonal antibody preparations) for the severe disease have also been disappointing. Some investigators have suggested repurposing drugs that are already licensed for use against other diseases. ${ }^{2}$ Some of these drugs target the host response to infection, not the virus itself. One of these drugs is dexamethasone, which improves survival in COVID-19 patients who require oxygen treatment but not in those who do not require oxygen. ${ }^{3}$

Repurposing cardiovascular drugs has attracted interest because many of these drugs (including statins) affect inflammation and endothelial dysfunction. ${ }^{4}$ Experts recommend continuing statin treatment in COVID-19 patients who are already being treated. ${ }^{5}$ This recommendation is based largely on numerous observational studies that suggest continuing treatment is safe. Nonetheless, most of these studies are based on outpatient-documented treatment. Because investigators seldom (if ever) consider the effects of statin withdrawal after hospital admission, their estimates of statin effectiveness are likely to be imprecise. It is unclear whether they recognize that statin withdrawal could be a problem.

\section{MATERIALS AND METHODS}

The study is based on a PubMed search conducted on July 24, 2021 using the search terms "statin withdrawal" (510 references) and "COVID-19, statins" (255 references). It is not a meta-analysis or systematic review. Instead, each article that reported data on statin treatment of COVID-19 patients was carefully read to determine whether the text, tables, figures, and citations contained

Abbreviations: ACEI, ACE inhibitor; ARB, angiotensin receptor blocker.

This is an open access article under the terms of the Creative Commons Attribution-NonCommercial-NoDerivs License, which permits use and distribution in any medium, provided the original work is properly cited, the use is non-commercial and no modifications or adaptations are made. (c) 2021 The Authors. Pharmacology Research \& Perspectives published by John Wiley \& Sons Ltd, British Pharmacological Society and American Society for Pharmacology and Experimental Therapeutics. 
evidence that the authors were aware of the clinical consequences of statin withdrawal. Citations for these COVID-19 articles and for statin withdrawal are available as supplementary material.

\section{RESULTS}

At least 42 individual observational studies and 14 meta-analyses evaluated the effectiveness of outpatient statin treatment on the outcomes of COVID-19 patients admitted to the hospital (supplementary material). These studies used a variety of methods to reach different conclusions about the effectiveness of treatment. Twenty-three reports concluded that statins reduced the occurrence of severe disease (e.g., ICU admission, mortality). ${ }^{\text {S1-S23 }}$ Thirteen reports concluded that statins might not be effective, but at least they were not harmful. ${ }^{\text {\$24-\$36 }}$ Six others concluded that statin treatment was actually associated with harm. ${ }^{\text {S37-542 }}$ Fourteen meta-analyses of these studies (based largely on outpatient information) provided varying estimates of statin effectiveness. ${ }^{\text {S43-556 }}$ In contrast, 11 observational studies uniformly showed that inhospital statin treatment was effective in reducing COVID-19 mortality (Table 1). ${ }^{\text {S57-S67 }}$

None of the 42 individual studies based on outpatientdocumented treatment ${ }^{\mathrm{S1-S42}}$ mentioned the consequences of statin withdrawal after hospital admission. Moreover, none of the 14 meta-analyses ${ }^{\mathrm{S43-555}}$ mentioned the problem of statin withdrawal, although several included information on better outcomes with inpatient compared with outpatient treatment. ${ }^{\$ 50,553,555,556}$ Only one of the 11 studies of in-hospital statin treatment ${ }^{6, a l s o ~} 565$ cited any references on the negative consequences of statin withdrawal.

\section{DISCUSSION}

The difference between the conflicting findings of outpatientdocumented statin treatment and uniform findings that inpatient treatment reduces COVID-19 severity and mortality is critically important. Documentation of statin treatment based only on outpatient information does not take into account the effects of statin withdrawal after hospital admission. Moreover, if inpatients are treated with statins, treatment might be withdrawn if they are transferred to ICUs, although intravenously administered statins are licensed if not widely available. ${ }^{7}$ Whenever statins are withdrawn, their beneficial effects on the host response can be rapidly lost. ${ }^{8}$ For example, cardiovascular investigators who studied patients hospitalized with acute myocardial infarction 15-20 years ago found that those who had been treated with statins as outpatients and whose statins were continued after hospital admission had lower mortality rates than those who had never received statins. ${ }^{9}$ The same benefit was seen in those who were started on statin treatment after hospital admission. However, those who had been treated with statins as outpatients but whose treatment was withdrawn after hospital

TABLE 1 The effects of in-hospital statin treatment and statin withdrawal on 28-30-day COVID-19 mortality

\begin{tabular}{|c|c|c|c|c|}
\hline \multicolumn{2}{|l|}{ Study } & \multicolumn{3}{|c|}{ Adjusted } \\
\hline Zhang (S57) & $\operatorname{PSM}(4: 1)-C C S$ & 0.58 & $0.43-0.80$ & 0.001 \\
\hline Rodriguez-Nava (S58) & Cohort, ICU & 0.38 & $0.18-0.77$ & 0.008 \\
\hline Mallow (S59) & Cohort & 0.54 & $0.49-0.60$ & $<0.001$ \\
\hline Masana (S61) & GM (1:1) & 0.60 & $0.39-0.92$ & 0.020 \\
\hline Fan (S62) & PSM, cohort & 0.25 & $0.07-0.92$ & 0.037 \\
\hline Torres-Pena (9, S63) & PSM, statins continued versus withdrawal & 0.67 & $0.54-0.84$ & $<0.001$ \\
\hline Memel $(10,564)$ & Marginal structural Cox model, IPTW & 0.57 & $0.37-0.86$ & 0.008 \\
\hline Lohia (S67) & PSM (1:1), cohort & 0.47 & $0.32-0.70$ & $<0.001$ \\
\hline
\end{tabular}

Abbreviations: CCS, case-control study; Cl, confidence interval; GM, genetics-matched; HR, hazard ratio; ICU, intensive care unit; IPTW, inverse probability treatment weighted; OR, odds ratio; PSM, propensity score-matched.

${ }^{a}$ The PS matched IPTW cohort analysis included demographic and comorbidity factors, clinical and laboratory test values, and the use of ACE inhibitors and angiotensin receptor blockers.

${ }^{\mathrm{b}}$ Statin treatment continued after hospital admission versus statin withdrawal; conditional logistic regression.

'Statin treatment continued after hospital admission versus statin withdrawal; marginal structural Cox model. 
admission had greater mortality than what was seen in never-treated patients.

These studies by cardiovascular investigators suggest that the effectiveness of statin treatment on the severity and mortality of COVID-19 can be accurately determined only by evaluating inhospital treatment. This has been confirmed in two studies that compared continuing versus not continuing statin treatment after hospital admission (Table 1). ${ }^{10,11}$

Remarkably, all of the 42 individual studies based on outpatientdocumented statin treatment failed to mention the possible effects of statin withdrawal after hospital admission. For example, in one study, outpatient statin treatment was associated with decreased mortality, but only $77 \%$ of outpatient-treated patients continued statin treatment after hospital admission. ${ }^{12}$ The investigators concluded that statin treatment was protective, but this was likely an underestimate because statins were withdrawn in $23 \%$ of hospitalized patients. In another study of 247 statin-treated patients, $46 \%$ of treatments were initiated after hospital admission and yet $29 \%$ of all statin treatments were later discontinued because of elevated liver function or creatine kinase tests. ${ }^{13}$

The 14 meta-analyses of statin treatment of COVID-19 were based largely on individual studies of out-patient-documented treatment. The individual studies must regarded as imprecise because they failed to consider the effects of statin withdrawal. For this reason, the point estimates reported in the meta-analyses must likewise be considered imprecise.

This is not to say that outpatient statin treatment is unimportant. In most studies reporting the effectiveness of outpatientdocumented statin treatment, unknown numbers of patients continued to be treated after hospital admission. Outpatient statins might also reduce the likelihood of developing symptomatic COVID-19 14 and might even prevent the development of "long COVID." Although clinical findings clearly indicate that statin treatment should be continued in COVID-19 patients with cardiovascular diseases after hospital admission, ${ }^{5}$ no study has yet shown that statins should be used to treat all hospitalized COVID-19 patients.

Cardiologists have known for 15-20 years that combination treatment with a statin and an ACE inhibitor (ACEI) or an angiotensin receptor blocker (ARB) is more effective than treatment with any of these agents alone. ${ }^{4,15}$ In COVID-19 patients, a propensity scorematched case-control study has recently shown that combination treatment with a statin and an ACEI or an ARB was more effective than single-agent treatment; combination treatment was associated with a 3-fold reduction in the odds of 28-day hospital mortality (OR $=0.33,95 \%$ C.I. 0.17-0.69, $p=.002)^{6}{ }^{6}$

There is a solid scientific and clinical rationale for repurposing statins and other widely available, inexpensive generic drugs to treat the host response to COVID-19 and other pandemic diseases. ${ }^{2,4}$ Unfortunately, a study published in late 2020 indicated that only $7 \%$ of the prospective clinical trials of COVID-19 treatments listed on ClinicalTrials.gov were focused on these drugs. ${ }^{16}$ Most of them were single-center studies and only a few were evaluating statins, ACE inhibitors, or ARBs. To the best of my knowledge, no randomized controlled trial of combination treatment has been planned or is underway. In the absence of clinical trials, physicians may have to rely on the findings of observational studies alone. ${ }^{17}$

\section{5 | CONCLUSION}

Any study that seeks to determine the effectiveness of statin treatment for COVID-19 patients (either by itself or in combination with other drugs) must consider the potentially negative consequences of statin withdrawal after hospital admission.

\section{ACKNOWLEDGEMENT}

This research did not receive any specific grant from funding agencies in the public, commercial, or not-for-profit sectors.

\section{DISCLOSURE}

The author declares no conflicts of interest.

\section{ORCID}

David S. Fedson (D) https://orcid.org/0000-0001-9963-1934

\section{REFERENCES}

1. Libby P, Luscher T. COVID-19 is, in the end, an endothelial disease. Eur Heart J. 2020;41:3038-3044. doi:10.1093/eurheartj/ehaa623

2. Martin JH, Head R. A pharmacological framework for integrating treating the host, drug repurposing and the damage response framework in COVID-19. Br J Clin Pharmacol. 2021;87:875-885. doi:10.1111/bcp.14551

3. Jensen MP, George M, Gilroy D, Sofat R. Beyond dexamethasone, emerging immuno-thrombotic therapies for COVID-19. Br J Clin Pharmacol. 2021;87:845-857. doi:10.1111/bcp.14540

4. Fedson DS. Treating the host response to emerging virus diseases: lessons learned from sepsis, pneumonia, influenza and Ebola. Ann TransI Med. 2016;4:421. doi:10.21037/atm.2016.11.03

5. Iqbal Z, Ho JH, Adam S, et al. Managing hyperlipidaemia in patients with COVID-19 and during its pandemic: an expert panel position statement from HEART UK. Atherosclerosis. 2020;313:126-136. doi:10.1016/j.atherosclerosis.2020.09.008

6. Byttebier G, Belmans L, Alexander M, et al. Hospital mortality in COVID-19 patients in Belgium treated with statins, ACE inhibitors and ARBs. Hum Vaccin Immunother. 2021;17:2841-2850. doi:10.1080/21645515.2021.1920271

7. Korani S, Bahrami S, Korani M, Banach M, Johnston TP, Sahebkar A. Parenteral systems for statin delivery: a review. Lipids Health Dis. 2019;18(1). doi:10.1186/s12944-019-1139-8

8. Cubeddu LX, Seamon MJ. Statin withdrawal: clinical implications and molecular mechanisms. Pharmacotherapy. 2006;26:1288-1296. doi:10.1592/phco.26.9.1288

9. Fonarow GC, Wright RS, Spencer FA, et al. Effect of statin use within the first 24 hours of admission for acute myocardial infarction on early morbidity and mortality. Am J Cardiol. 2005;96:611616. doi:10.1016/j.amjcard.2005.04.029

10. Torres-Peña JD, Pérez-Belmonte LM, Fuentes-Jiménez F, et al. Prior treatment with statins is associated with improved outcomes of patients with COVID-19: data from the SEMI-COVID-19 Registry. Drugs. 2021;81:685-695. doi:10.1007/s40265-021-01498-x

11. Memel ZN, Lee JJ, Foulkes AS, Chung RT, Thaweethai T, Bloom PP. Statins are associated with improved 28-day mortality in patients hospitalized with SARS-CoV-2 infection. medRxiv. 2021.03.27.21254373. doi: 10.1101/2021.03.27.21254373 
12. Gupta A, Madhavan MV, Poterucha TJ, et al. Association between antecedent statin use and decreased mortality in hospitalized patients with COVID-19. Nat Commun. 2021;12:1325. doi:10.1038/ s41467-021-21553-1

13. McCarthy CP, Murphy S, Jones-O'Connor M, et al. Early clinical and sociodemographic experience with patients hospitalized with COVID-19 at a large American healthcare system. EClinicalMedicine. 2020;26:100504. doi:10.1016/j.eclinm.2020.100504

14. De Spiegeleer A, Bronselaer A, Teo JT, et al. The effects of ARBs, ACEis, and statins on clinical outcomes of COVID-19 infection among nursing home residents. J Am Med Dir Assoc. 2020;21(7):909914.e2. doi:10.1016/j.jamda.2020.06.018

15. Koh KK, Sakuma I, Shimada K, Hayashi T, Quon MJ. Combining potent statin therapy with other drugs to optimize simultaneous cardiovascular and metabolic benefits while minimizing adverse events. Korean Circ J. 2017;47:432-439. doi:10.4070/kcj.2016.0406

16. Varshney AS, Wang DE, Bhatt AS, et al. Characteristics of clinical trials evaluating cardiovascular therapies for Coronavirus Disease
2019 registered on ClinicalTrials.gov: a cross-sectional analysis. Am Heart J. 2021;232:105-115. doi:10.1016/j.ahj.2020.10.065

17. Concato J, Shah N, Horwitz R. Randomized, controlled trials, observational studies, and the hierarchy of research designs. N Engl J Med. 2000;342:1887-1892. doi:10.1056/NEJM200006223422507

\section{SUPPORTING INFORMATION}

Additional Supporting Information may be found online in the Supporting Information section.

How to cite this article: Fedson DS. Statin withdrawal and treating COVID-19 patients. Pharmacol Res Perspect. 2021;9:e00861. https://doi.org/10.1002/prp2.861 